\title{
Long-Term Adaptation of Lung Tumor Cell Lines with Increasing Concentrations of Nitric Oxide Donor
}

\author{
James A. Radosevich ${ }^{*, 1,2}$, Kim M. Elseth ${ }^{1,2}$, Benjamin J. Vesper ${ }^{1,2}$, Gabor Tarjan $^{3}$ and \\ G. Kenneth Haines III $^{4}$ \\ ${ }^{I}$ Center for Molecular Biology of Oral Diseases, College of Dentistry, University of Illinois at Chicago, Chicago, IL \\ 60612, USA \\ ${ }^{2}$ Jesse Brown VAMC, Chicago, IL 60612, USA \\ ${ }^{3}$ Department of Pathology, John H. Stroger, Jr. Hospital of Cook County, Chicago, IL 60612, USA \\ ${ }^{4}$ Department of Pathology, Yale University School of Medicine; New Haven, CT 06510, USA
}

\begin{abstract}
The free radical nitric oxide (NO) is known to play an important role in the biology of human cancers, including lung cancer. However, it is still not clear how elevated amounts of nitric oxide affect tumor development and propagation. Herein we develop an in vitro model system to study these effects in lung tumor cells. Two cell lines-one human lung adenocarcinoma (A549) and one mouse adenocarcinoma (LP07) cell line-were adaptively grown in increasing concentrations of the NO donor DETA-NONOate over several months. Both cell lines were successfully adapted to high levels of NO (HNO). Experiments validated the adaptation occurred as a result of the exogenous NO produced by the DETA-NONOate, and was not merely a response to the chemical composition of DETA-NONOate. No morphological differences were observed between cells that were adapted to the HNO and cells which did not undergo the adaptation process (i.e., "parent cells"). Parent cells were unable to survive when placed directly in media containing high levels of DETA-NONOate, suggesting that the adapted cells underwent a biological change enabling them to survive and grow in a HNO environment. The adapted cells were found to grow faster than the parent cells under both normal growth conditions and stressful growth conditions (serum-less media, growth on soft agar) even when the DETA-NONOate was removed from the HNO culture media. These adapted cell lines can serve as a novel tool for use in future experiments designed to better understand the role nitric oxide plays in lung cancer.
\end{abstract}

Keywords: Adenocarcinoma, nitric oxide, nitric oxide synthase (NOS), lung cancer, nitrogen and oxygen reactive species, cellular adaptation.

\section{INTRODUCTION}

Despite extensive research, lung cancer remains the leading cause of cancer death in the world. It is estimated that over 215,000 new cases of lung cancer were diagnosed in the United States in 2008, and $29 \%$ of U.S. cancer deaths in 2008 resulted from lung cancer [1]. Oxidative/reductive (redox) DNA damage is thought to be a significant contributor in the progression of cancer [2,3], and one redox species in particular, nitric oxide (NO), has been implicated in a number of physiological and pathophysiological processes in a variety of human tissues [4]. NO and a number of its metabolic byproducts are involved in two completely opposite functions: they can serve as either protective agents against malignancies, or as antagonists which can promote carcinoma formation and development [5]. In the case of the former, $\mathrm{NO}$ is produced as both an antimicrobial and an anti-tumor immune response, while in the case of the latter, unregulated and prolonged NO expression can induce mutational events that may lead to cancer $[6,7]$.

*Address correspondence to this author at the Center for Molecular Biology of Oral Diseases, University of Illinois at Chicago, College of Dentistry, 801 S. Paulina St., Chicago, IL 60612, USA; Tel: (312) 996-9538; Fax: (312) 996-9592; E-mail: jrados@uic.edu
Nitric oxide is produced from the amino acid L-arginine by a family of isoenzymes called nitric oxide synthase (NOS) $[8,9]$. There are three isoforms of NOS, defined according to their activity or the tissue type in which they were first described: endothelial constitutive NOS (ecNOS), inducible NOS (iNOS), and neuronal NOS (nNOS) [10]. Our laboratory has investigated NOS and nitrotyrosine (a marker of NOS activity) expression levels in a variety of carcinomas, including head and neck [4,11], salivary [12], and esophageal tumors [13]. Over-expression of at least one NOS isoform was observed for each tumor type tested, relative to normal tissue, and nitrotyrosine over-expression was observed in the head and neck tumors, the only tumor type we tested for nitrotyrosine expression.

To date, however, there is still uncertainty regarding the role of NO and NOS in lung adenocarcinomas. While a small number of studies have failed to detect a difference in NOS expression between lung tumor cells and normal cells $[14,15]$, the majority of work in this area has found that levels of NOS (particularly iNOS) and/or nitrotyrosine are higher in lung carcinomas than in normal tissue [15-19]. Furthermore, a recent study found a correlation between smoking - a known source of high levels of reactive nitrogen and oxygen species - and increased expression of iNOS: 
lung tumor tissues taken from smokers exhibited higher levels of iNOS than lung tumor tissues taken from nonsmokers [20]. The patient population in this study was controlled to study only smokers versus nonsmokers; all patient properties and tumor characteristics (including age and sex of patient, tumor type and stage, etc.) were effectively controlled. The spectrum of NOS expression could be linked to the biological behavior of the tumors, therefore suggesting NOS expression could be used to predict patient outcome. The results of this work suggest that differences in NOS- and NO-related gene products are fundamental properties of these tumors. There are a number of clinical issues that need to be addressed: 1) It is currently unclear how such differences in NO biology affect lung tumor carcinogenesis, 2) It is unclear if the treatment of tumors influences NO expression, and 3) It is also unknown if tumors with varying expression levels of $\mathrm{NO}$ necessitates different therapeutic approaches. Currently, there is no reasonable way to test in humans all of the possible parameters involved with this spectrum of NO expression and its relationship to the biology of the tumors. Therefore, a model system that represents the spectrum of NO expression in tumors would be of considerable value to test and determine a wide spectrum of biological properties related to NO tumor biology.

In an effort to better comprehend the underlying biological effects of NO exposure in lung cancer, we herein develop a model cell line system to study human and mouse lung tumors "adapted" to increasing levels of nitric oxide over a long period of time. Previous in vitro studies of NO exposure to lung tumor cells, including those performed by our laboratory $[21,22]$, have focused exclusively on the NO donor being delivered in a short time frame (typically no longer than 72 hours). In contrast, the system developed herein sought to expose cells to a slow, gradually, increasing concentration of NO donor over an extended period of time, with the belief that these cells would eventually sustain longterm viability in a high-free radical environment that would otherwise be toxic to cells that had not undergone adaptation. Successfully creating these adapted cell lines would result in a practical model system to study the mechanisms by which cells differentially respond to varying degrees of nitrosive stress, as seen in vivo.

\section{MATERIALS AND METHODOLOGY}

\section{Cell Culture and Cell Lines}

All media and supplements were purchased from Invitrogen (California, USA), except where noted. A549, a human lung adenocarcinoma cell line [23,24], and LP07, a mouse lung adenocarcinoma cell line [25-27], were used in this study. The A549 cell line was purchased from American Type Culture Collection (Virginia, USA); the LP07 cell line was previously obtained as a generous gift from Dr. Slobodanka Klein at the University of Buenos Aires, Argentina. A549 was grown in RPMI-1640 media, and LP07 was grown in MEM media. All media was supplemented with $10 \%$ fetal calf serum inactivated at $56^{\circ} \mathrm{C}$ for 30 minutes, $100 \mathrm{U} / \mathrm{mL}$ penicillin, $100 \mu \mathrm{g} / \mathrm{mL}$ streptomycin, $2 \mathrm{mM} \mathrm{L}-$ glutamine, and $2.5 \mu \mathrm{g} / \mathrm{mL}$ Amphotericin B solution. The MEM media was additionally supplemented with $100 \mathrm{mM}$ MEM nonessential amino acids and $1 \mathrm{mM}$ sodium pyruvate
(CellGro, Inc., Virginia, USA). Cell lines were grown in a humidified incubator at $37{ }^{\circ} \mathrm{C}$ and $5 \% \mathrm{CO}_{2}$. All other reagents were obtained from Sigma-Aldrich (St. Louis, MO, USA) unless otherwise stated.

\section{Cell Adaptation Process}

Both A549 and LP07 were "adapted" to the nitric oxide donor (Z)-1-[2-(2-aminoethyl)-N-(2-ammonioethyl)amino] diazen-1-ium-1,2-diolate (DETA-NONOate). DETANONOate was purchased from Sigma Life Sciences (St. Louis, Missouri, USA) and used without further purification. Stock solutions of DETA-NON Oate were prepared in sterilized water and sterile filtered using 0.22 micron filter units. To begin the adaptation process, cells were passaged with trypsin-EDTA and transferred to a new flask containing media supplemented with $50 \mu \mathrm{M}$ DETA-NONOate. The flask was placed in the incubator and grown at $37^{\circ} \mathrm{C}$ and $5 \%$ $\mathrm{CO}_{2}$. After reaching $\sim 90 \%$ confluency, the cells were again passaged with trypsin-EDTA; the resulting cultures were grown in media treated with $75 \mu \mathrm{M}$ DETA-NONOate. This process was repeated, with the NO donor concentration being increased in 25 or $50 \mu \mathrm{M}$ increments, until the concentration of DETA-NONOate reached the highest desired level $(600 \mu \mathrm{M}$ for the A549 cells and $300 \mu \mathrm{M}$ for the LP07 cells). These end point concentrations were chosen on the basis that they were a known dose able to kill $100 \%$ of the cells in 24 hours, when the parent cell line was "shocked" (rather than adapted to) into media containing that level of DETA-NONOate. The ability to grow in a lethal dose of DETA-NONOate would imply that biological changes had taken place in such cells. As the adaptation process progressed, aliquots of the adapted cells were periodically removed for cryostorage.

Separate "parent" cells were maintained as controls. Parent cells were grown under standard conditions (i.e., no nitric oxide donor was added).

During both the adaptation process and the subsequent maintenance of the fully adapted cells, the cells were replenished with media containing DETA-NONOate every 2-3 days. Parent cells were also replenished with fresh media every 2-3 days.

\section{Verification of Adaptation End-Point}

Following adaptation of the cells lines to 300 and 600 $\mu \mathrm{M}$ DETA-NONOate for LP07 and A549, respectively, we sought to confirm that these adapted cells had been developed to withstand a HNO environment that would not support the growth of the corresponding parent cells. For each cell line, parent (A549 \& LP07) and adapted cells (A549-HNO \& LP07-HNO) were seeded $(100 \mu \mathrm{L})$ into 96well plates and grown for 24 hours, to $\sim 70 \%$ confluency. (LP07-HNO cells were grown in media containing $300 \mu \mathrm{M}$ DETA-NONOate; A549-HNO cells were grown in media containing $600 \mu \mathrm{M}$ DETA-NONOate.) The media was then removed, and $100 \mu \mathrm{L}$ of media without DETA-NONOate was added to the HNO cells, while $100 \mu \mathrm{L}$ of media containing either $300 \mu \mathrm{M}$ (LP07 cells) or $600 \mu \mathrm{M}$ (A549 cells) DETA-NONOate was added to the parent cells. Plates were then incubated for an additional 72 hours, at which time 3-(4,5-dimethylthiazol-2-yl)-2,5-diphenyltetrazo-lium bromide (MTT) cell proliferation/viability assays were 
performed. The assay was performed as follows: the media was removed, and $100 \mu \mathrm{L}$ of $2 \mathrm{mg} / \mathrm{mL}$ MTT (Sigma, St. Louis, Missouri, USA) in phosphate buffer saline (PBS) was added. Plates were incubated at $37^{\circ} \mathrm{C}$ for 5 hours, after which time the MTT was aspirated. The remaining purple formazan crystals were dissolved in $100 \mu \mathrm{L}$ of Dimethyl Sulfoxide (DMSO), and the absorbance of each well was read at 540 nm using a SpectraMax ${ }^{\circledR}$ Plus 384 spectrophotometer (Molecular Devices, Sunnyvale, California, USA). A minimum of three independent trials were carried out for each experiment, and each data point represents the average of at least four microtiter wells for each plate. Thus, a minimum of 12 independent values were measured for each cell line at each time point. Individual trials were normalized and averaged.

\section{Validation of Nitric Oxide Donor Source}

To confirm that the adapted cells were being affected by the NO production from the DETA-NONOate, and not the chemical makeup of the donor itself, adapted cells were grown in the presence of different NO donors. A549-HNO cells were seeded $(100 \mu \mathrm{L})$ into 96-well microtiter plates and grown in RPMI-1640 media containing $600 \mu \mathrm{M}$ DETANONOate for 24 hours, to $\sim 70 \%$ confluency. The media was then removed, and fresh media containing $600 \mu \mathrm{M}$ of one of the following donors was added: DETA-NONOate; (N)-[4[1-(3-aminopropyl)-2-hydroxy-2-nitroso-hydrazino]butyl]-1,3propanediamine (spermine-NONOate; Oxis International, Beverly Hills, CA, USA); ( \pm )-S-nitroso-N-acetylpenicillamine (SNAP; Sigma Life Sciences, St. Louis, Missouri, USA); or N-( $\beta$-D-glucopyranosyl)- $\mathrm{N}^{2}$-acetyl-S-nitroso-D,Lpenicillaminamide (glyco-SNAP; Oxis International, Beverly Hills, CA, USA). Stock solutions of spermine-NONOate, SNAP, and glyco-SNAP were prepared in sterilized distilled water and sterile filtered, analogous to the solution preparation of DETA-NONOate described above. The microtiter plates were then returned to the incubator, and MTT cell proliferation/viability assays were performed after 24, 48, and 72 hour exposure to the desired NO donor.

\section{$\mathrm{H}_{2} \mathrm{O}_{2}$ Exposure Growth Assays}

Parent and HNO adapted cells were seeded $(100 \mu \mathrm{L})$ into 96-well plates in the appropriate media and incubated overnight. In order to find the concentration range of hydrogen peroxide that the cells could tolerate, $0.1 \mathrm{~mL}$ of $30 \%$ hydrogen peroxide solution was added to first column of wells in a 96-well microtiter plate and diluted by 1:1 serial dilution. Then, the cells were incubated at $37{ }^{\circ} \mathrm{C}$ overnight and MTT assays were used to measure the cell viability of both parent and HNO adapted cell lines, as described above. The assays were repeated three times to verify the concentration ranges for each of the cell line pairs.

\section{Normal Media Growth Assays}

The MTT assay was used to measure cell proliferation/viability of the parent and HNO adapted cells under the growth conditions established in this study. Parent and HNO adapted cells were seeded into 96-well microtiter plates at $100 \mu \mathrm{L}$. Parent cells were seeded and grown in the presence of the appropriate media formulation (RPMI-1640 for A549; MEM for LP07). The HNO adapted cells were seeded and grown in media containing either $300 \mu \mathrm{M}$ (LP07-
HNO) or $600 \mu \mathrm{M}$ (A549-HNO) DETA-NONOate. MTT assays were carried out as described above, at the desired time points $(24,48$, or 72 hours after seeding).

\section{Serum-Less Growth Assays}

Parent and A549-HNO cells were seeded $(100 \mu \mathrm{L})$ into 96-well plates in the appropriate media (i.e., standard media for parent cell lines; media treated with $600 \mu \mathrm{M}$ DETANONOate for the adapted cells). The plates were incubated for 24 hours, after which time the media was replaced with analogous serum-less media (i.e., lacking the 10\% FCS). With the exception of the FCS, all other components of the media, including the additional DETA-NONOate for the HNO adapted cells, were identical to the original formulation used. Plates were then incubated for an additional 24, 48, and 72 hours. MTT assays were performed at each time point, as described above. LP07 and LP07-HNO cells were not studied in this assay format.

\section{Soft Agar Growth Assays}

The 96-well soft agar assay method was used to compare the growth properties of the A549 parent and A549-HNO, as well as LP07 and LP07-HNO; more malignant cells grow better on soft agar than do less malignant cells [28]. The soft agar mixture consisted of $50 \%$ low melting point agarose (Sigma A-9539, gelling temperature approximately $36^{\circ} \mathrm{C}$; Sigma, Missouri, USA) and 50\% 2x RPMI supplemented with $20 \%$ fetal calf serum, $200 \mathrm{U} / \mathrm{mL}$ penicillin, $200 \mu \mathrm{g} / \mathrm{mL}$ streptomycin, $4 \mathrm{mM}$ L-glutamine, and $5 \mathrm{mcg} / \mathrm{mL}$ Amphotericin B solution. After the agarose was autoclaved and incubated at $42{ }^{\circ} \mathrm{C}$ for at least 30 minutes, the agarose and 2x RPMI- 1640 were mixed and incubated at $42^{\circ} \mathrm{C}$ until used. The agarose mixture $(100 \mu \mathrm{L})$ was loaded into a 96well microtiter plate and allowed to harden. Parent and HNO adapted cells $(100 \mu \mathrm{L})$ were then added on top of the solidified agar. (Parent cells were added in standard $1 \mathrm{X}$ growth media; HNO adapted cells were added in standard $1 \mathrm{X}$ growth media supplemented with 300 or $600 \mu \mathrm{M}$ DETANONOate; LP07-HNO and A549-HNO respectively.) The plates were then incubated for 12 hours at $37^{\circ} \mathrm{C}$, enabling the cells to attach to the agar. Following incubation, the media above the agar was removed, and the plates were returned to the incubator for an additional 24,48 , or 72 hours. At the desired time point, MTT assays were performed: $100 \mu \mathrm{L}$ of 2 $\mathrm{mg} / \mathrm{mL}$ MTT in PBS was added, and the plates were incubated at $37^{\circ} \mathrm{C}$ for 5 hours. The MTT solution was then removed, and the remaining purple formazan crystals were dissolved in $100 \mu \mathrm{L}$ of DMSO. The resulting (purple) solution in each well was then transferred to a new 96-well plate; absorbance readings were obtained as described above.

\section{Data Analysis and Statistics}

For each experiment type reported herein, at least three independent experiments were conducted. A minimum of four wells were run in each experiment for a total of twelve wells. All values that were more than two standard deviations from the mean were not included in the final analysis. The independent experiment values were normalized with respect to each other and then averaged. The error bars reported represent one standard deviation from the averaged normalized mean. Data was graphed using SigmaPlot version 9.0 (Systat Software, Inc., Chicago, IL, USA). 


\section{RESULTS}

\section{Cell Adaptation}

Prior to beginning the adaptation process, it was necessary to first select an appropriate nitric oxide donor. Previous work from our laboratory analyzed the kinetics of four commonly used nitric oxide donors: DETA-NONOate, SpermineNONOate, SNAP, and glyco-SNAP [22]. Anticipating that the adaptation process would be a long endeavor, we sought a donor with a long half-life (and therefore slow decay rate), as this would result in fewer required replenishments of the donor. Among the four donors, DETA-NONOate possesses the longest half-life (approximately $24 \mathrm{hrs}$ at $37^{\circ} \mathrm{C}$ and $\mathrm{pH}$ 7.4) and was therefore selected for the current study. Furthermore, the NONOate donors (including both DETA-NONOate and spermine-NONOate) donate $\mathrm{NO}$ as a free radical in aqueous solution, at a rate of two moles of NO per mole of DETANONOate. In contrast, the thiol-based donors (SNAP and glyco-SNAP) contribute only 1 mole of NO per mole of donor and do so as a nitrosonium species $\left(\mathrm{NO}^{+}\right)$to other thiols. The combination of the long half-life, mode of delivery, and rate of delivery of DETA-NONOate allowed for the maximum addition of $\mathrm{NO}$ to the cells at a minimum number of applications.

It is important to emphasize that the concentration referred to here is the concentration of the NO donor, and not the actual concentration of NO produced by the donor to which the cells are exposed. The actual concentration of NO delivered by the donor fluctuates based upon the donor's half-life, age of the stock solution, and the time that it was used in the cell culture incubations. Thus, expressing the concentration in terms of the amount of donor added to the cells provides a more convenient way to express the amount of NO applied to the cells.
A549, a human lung adenocarcinoma cell line, and LP07, a mouse adenocarcinoma cell line, were selected to undergo adaptation. To begin the process, the tumor cells were passaged and added to a new flask containing media supplemented with $50 \mu \mathrm{M}$ DETA-NONOate. The NO donor was added to the media immediately prior to the cells being introduced to the media. Cell growth was initially slowed by the presence of the NO donor, but the cells eventually recovered and were able to grow robustly. The cells were split at each incremental increase of DETA-NONOate, where upon one flask was maintained at the current concentration of donor, and a separate flask was used for the next higher concentration. The adaptation process continued as the amount of DETA-NONOate added to the media was increased gradually in 25 or $50 \mu \mathrm{M}$ increments (see Fig. 1).

Each data point on the growth curves represents the addition of fresh DETA-NONOate to the cells, either as a replenishment of media or a cell passage event. As noted above, slowed growth often occurred immediately after the cell lines were exposed to a higher dose of NO donor. Cell death (up to $\sim 50 \%$ ) was sometimes observed, but the cells were typically able to recover and eventually grew to confluency. In cases where cell death was particularly high upon addition of the next increment of DETA-NONOate, the media was replaced with fresh media containing a lesser amount of donor (typically $25 \mu \mathrm{M}$ less), giving the cells a chance to recover from the newly introduced level of DETANONOate.

Attempts were made to adapt each cell line to a maximum concentration of $600 \mu \mathrm{M}$ DETA-NONOate. (The $600 \mu \mathrm{M}$ plateau was chosen for reasons described in the following section, Verification of Adaptation End-Point.) The A549 lung adenocarcinoma cell line adapted quickly,

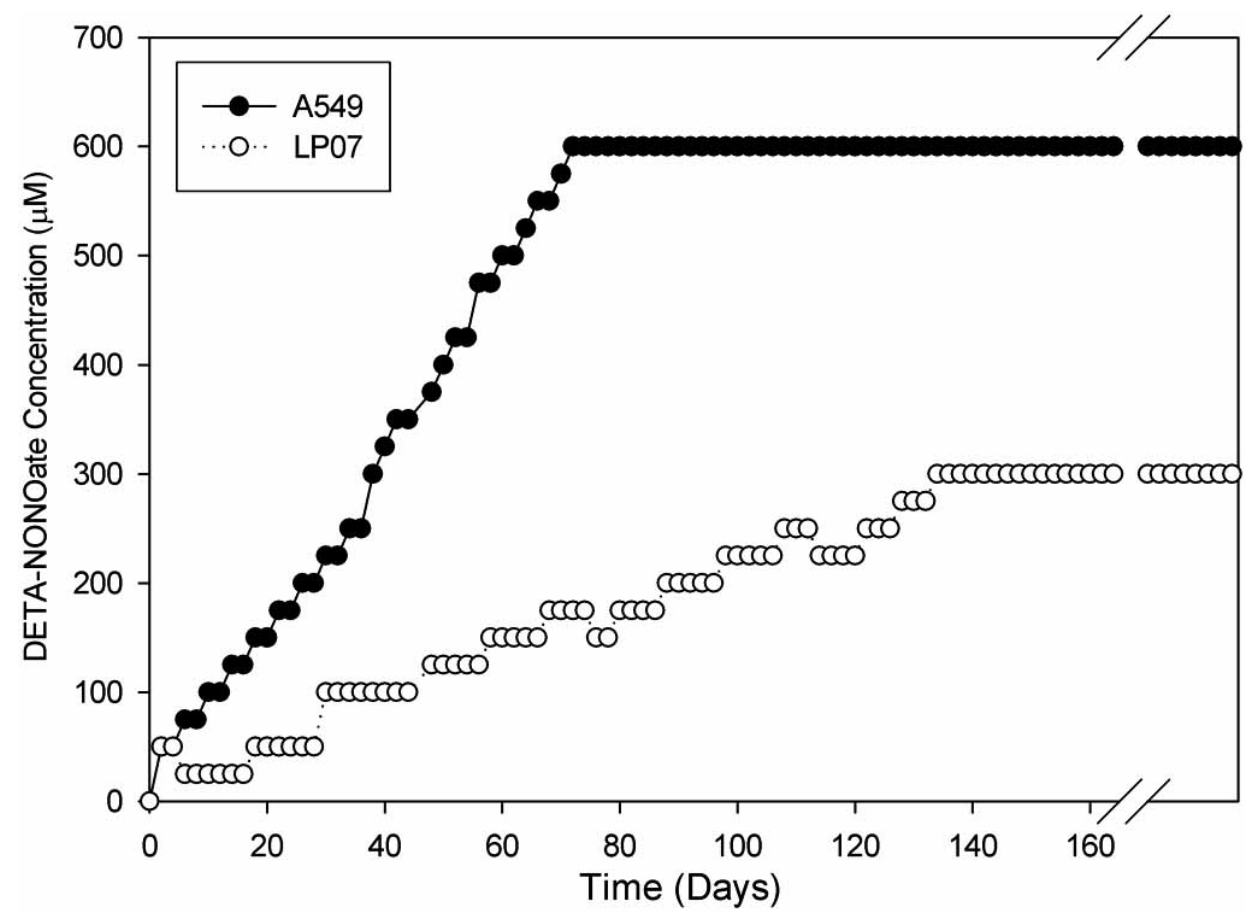

Fig. (1). Adaptation curves of the A549 and LP07 cell lines. Each data point represents the addition of fresh DETA-NONOate, either as a passaging event or a replenishment of media. Cells were maintained at $300 \mu \mathrm{M}$ (LP07) or $600 \mu \mathrm{M}$ (A549) DETA-NONOate for over one year; therefore, we speculate these cell lines are immortalized and could theoretically grow to infinity. 
reaching $600 \mu \mathrm{M}$ in approximately 65 days (Fig. 1). The cells were able to thrive with each subsequent DETANONOate addition; at no time during the adaptation did the concentration have to be reduced.

In stark contrast, the mouse tumor cell line, LP07, adapted very slowly. The adaptation process was stopped upon reaching $300 \mu \mathrm{M}$ DETA-NONOate (Fig. 1), as the adaptation time for the LP07 cell line was greater than double that of the A549 cells. It took approximately 135 days for the cells to reach $300 \mu \mathrm{M}$ DETA-NONOate. High levels of cellular toxicity were observed with this cell line when increased NO donor concentrations were attempted, making it frequently necessary to reduce the DETANONOate concentration during the adaptation process.

During the adaptation process and upon reaching the adaptation plateau (i.e., $600 \mu \mathrm{M}$ for $\mathrm{A} 549 ; 300 \mu \mathrm{M}$ for LP07), the media containing the NO donor was replenished every 2-3 days. Given the decay rate of DETA-NONOate under the incubation conditions (approximately $24 \mathrm{hrs}$ at $37^{\circ} \mathrm{C}$ and $\mathrm{pH} 7.4$ ), the amount of NO available to the cells was greatest immediately upon addition of the $\mathrm{NO}$ donor and decreased thereafter, according to its half-life, until the media was replaced with fresh DETA-NONOate. The periodic addition of NO donor every 2-3 days thus resulted in a cyclical fluctuation of the amount of $\mathrm{NO}$ available to the cells at any one given time.

We note that during the adaptation process, fresh stock solutions of DETA-NONOate were prepared immediately prior to adding the NO donor to the media and treating the cells. However, after the A549-HNO cells had reached full adaptation, larger volumes of RPMI-1640 media containing $600 \mu \mathrm{M}$ DETA-NONOate were sometimes prepared in advance. These "pre-made" DETA-NONOate/media solutions were stored at $4^{\circ} \mathrm{C}$ for periods of no longer than two weeks prior to introduction into the cell population. The A549HNO cells showed no difference in viability and propagation rates when grown in the two-week old DETA-NONOate/ media solution, versus being grown in a freshly prepared solution [data not shown].

\section{Verification of Adaptation End-Point}

To determine that the HNO cells had been exposed to concentrations of nitric oxide high enough to significantly alter the cellular biology of the cells, pilot studies were conducted in which A549 parent cells were exposed to various concentrations of DETA-NONOate. From these studies, $600 \mu \mathrm{M}$ DETA-NONOate was found to consistently kill all of the parent cells after 24 hours [data not shown]. Thus, $600 \mu \mathrm{M}$ was chosen as the plateau target level in the adaptation process, as cells which could be adapted to this level would have to had changed their biological constitution.

To verify the chosen end-point, the A549-HNO cells were grown in media in which the NO donor was removed; the parent A549 cells were grown in media containing 600 $\mu \mathrm{M}$ DETA-NONOate. As expected, the parent cells were not able to survive when placed directly into a HNO environment of $600 \mu \mathrm{M}$ donor [data not shown]. Removal of the NO donor source from A549-HNO cells did not affect growth over the 72 hour period measured. Analogous results were obtained for the LP07 and LP07-HNO cell lines at a DETA-NONOate concentration of $300 \mu \mathrm{M}$.

\section{Validation of Nitric Oxide Donor Source}

As described above, DETA-NONOate was chosen as the nitric oxide donor for the cell line adaptations due to its long half-life, donor delivery, and rate properties. To ensure that the cells were adapting to the NO being produced in the presence of the donor, and not from inherent toxicity of the donor backbone, A549-HNO cells were grown in the presence of four different donors: DETA-NONOate, spermineNONOate, SNAP, and glyco-SNAP. Fig. (2) shows the growth curves of A549-HNO cells exposed to $600 \mu \mathrm{M}$ of the four donors for up to 72 hours. While cell viability was higher for the two thiol-based donors (SNAP and glycoSNAP), the adapted cells were able to survive and grow in

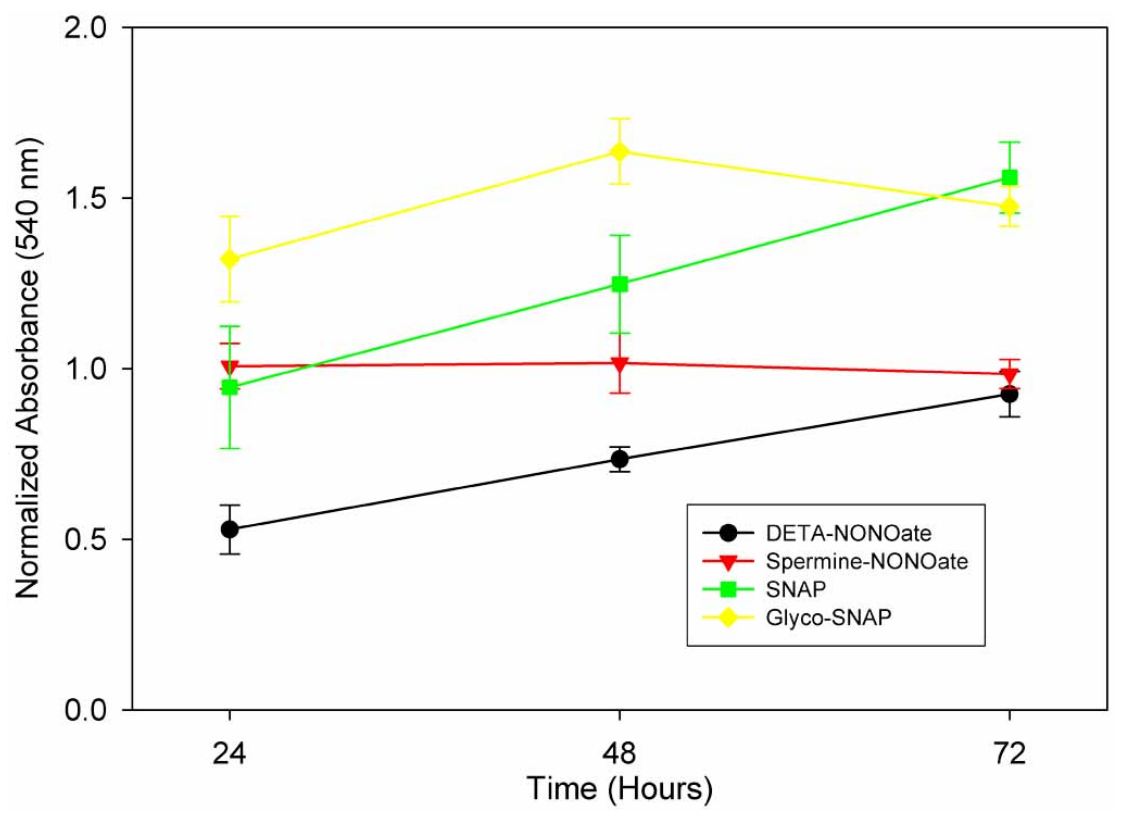

Fig. (2). MTT proliferation/viability curves of A549-HNO cells exposed to various NO donors at $600 \mu \mathrm{M}$ for 72 hours. 
the presence of all four donors over the course of 72 hours.

\section{$\mathrm{H}_{2} \mathrm{O}_{2}$ Growth Assays}

In order to determine if the HNO cells were able to survive a high free radical environment generated by an oxygen-based donor, A549 and LP07 parent and HNO cells were exposed to varying levels of $\mathrm{H}_{2} \mathrm{O}_{2}$. Dose-dependent growth curves are shown in Fig. (3). In the A549 cell lines, both A549 parent and A549-HNO cells were killed at concentrations of $0.055 \mathrm{mM} \mathrm{H} \mathrm{H}_{2}$ and above. However, at concentrations of $0.028 \mathrm{mM}$ and below, the A549-HNO cells exhibited far greater viability/proliferation than their corresponding parent cells. In contrast, the LP07 and LP07HNO cells exhibited similar viability/proliferation at all concentrations of $\mathrm{H}_{2} \mathrm{O}_{2}$ tested.

\section{Growth Curves}

Growth curves of A549, LP07, and the HNO cell lines were carried out in normal media and on soft agar; growth curves of the parent A549 and A549-HNO were also carried out in serum-less media. Under normal growth conditions, the HNO cells (for both human A549-HNO and mouse LP07-HNO), grown in media supplemented with either 300 (LP07) or 600 (A549) $\mu \mathrm{M}$ DETA-NONOate, were found to grow faster than their corresponding parent cells grown in media without NO donor (Fig. 4).

Growth curves of the parent and adapted cells were also compared under two stressful conditions: serum-less media (Fig. 5) and growth on soft agar (Fig. 6). In both cases, the
A549-HNO cells again grew at a measurably faster rate than their corresponding parent cells. Similar results were observed for the LP07-HNO cells grown on soft agar. LP07 and LP07-HNO were not tested under serum-less growth conditions.

\section{DISCUSSION}

We have found that both human and mouse lung adenocarcinoma cell lines can be incrementally adapted to comparatively high levels of nitric oxide exposure, over long periods of time (Fig. 1). The adaptation process differed between the human and mouse cell lines in both the maximum concentration of NO that the cells were able to withstand and the amount of time it took for the adaptation process to occur. In both cell lines however, accelerated growth properties were observed for the HNO cells versus the parent cells under all growth conditions tested: normal media, serum-less media, and growth on soft agar (Figs. 46). This accelerated growth was not due to the NO per se, as $\mathrm{NO}$ in lower concentrations is known to stimulate cell growth, because the HNO cell lines retained their growth properties even in the absence of the added NO donor (data not shown).

Despite the relative similarities in the growth behavior of the HNO cell lines versus the corresponding parent cell line, significant differences were observed in the kinetics of adaptation between the A549 human cells and the LP07 mouse cells. The A549 cells were able to easily withstand the increased DETA-NONOate additions, without the need

\section{A549}
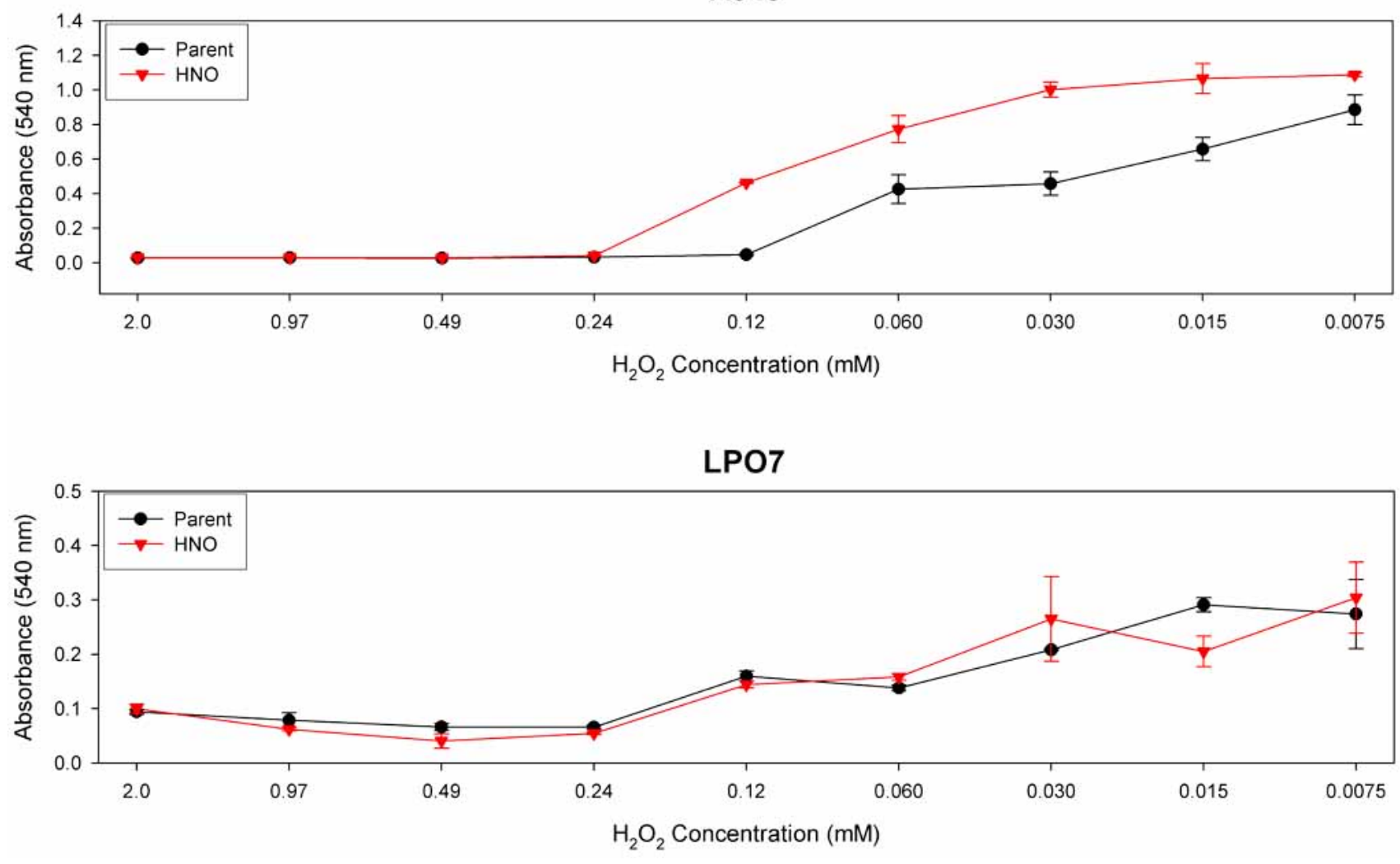

Fig. (3). MTT proliferation/viability assays of A549 and LP07 parent and HNO cells in response to a hydrogen peroxide dilution series. The media of the HNO cells was supplemented with $300 \mu \mathrm{M}$ (LP07-HNO) or $600 \mu \mathrm{M}$ (A549-HNO) DETA-NONOate. 

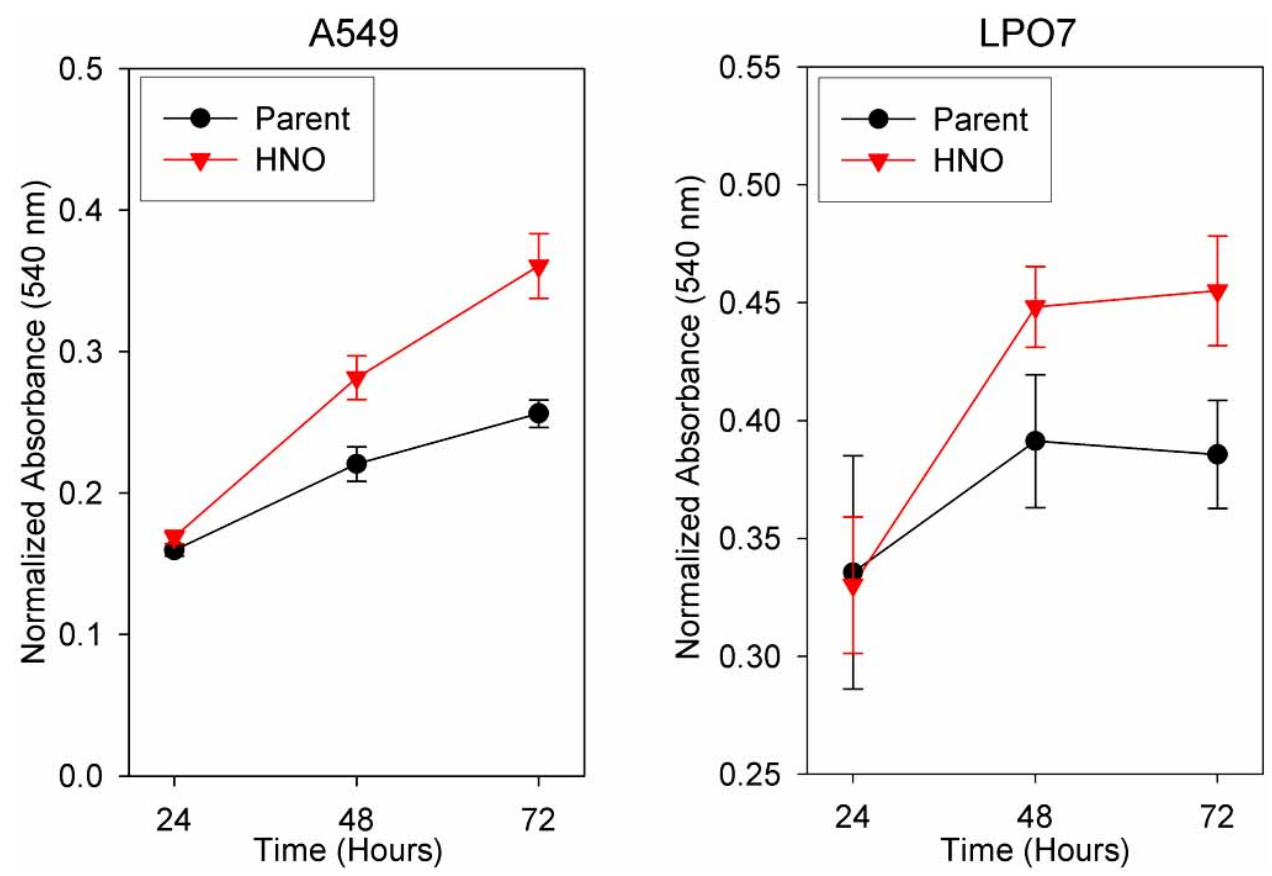

Fig. (4). MTT proliferation/viability curves of A549 and LP07 parent and HNO cells in standard growth media. The media of the HNO cells was supplemented with $300 \mu \mathrm{M}$ (LP07-HNO) or $600 \mu \mathrm{M}$ (A549-HNO) DETA-NONOate.

to reduce the concentration at any point during the adaptation process, and were able to grow robustly in the resulting HNO environment. In contrast, the LP07 cells had difficulty adapting to increased doses of NO donor. These cells were stopped at $300 \mu \mathrm{M}$ in part due to extremely slow adaptation, but also because this concentration was lethal to the parent. Furthermore, during the adaptation of the LP07 cell line, it was frequently necessary to reduce the concentration of DETA-NONOate in order for the cells to survive the increased free radical environment. At this time it is unclear why the A549 cell line was able to adapt more quickly than the LP07 cell line. The differences between these two cell lines reflects what is found clinically; some patients' tumors progress rapidly regardless of the treatment plan, while other patients have a slower, more indolent course.

While it is thought that the A549 cell line could be adapted to levels of DETA-NONOate greater than $600 \mu \mathrm{M}$, we do not believe that it is necessary to pursue such donor concentrations. For both the A549-HNO and LP07-HNO cell lines, the end-point verification studies confirmed that the HNO cells can survive in a harsh, HNO environment that is not viable for the parent cells. These results suggest that the HNO adapted cells have undergone molecular changes and now represent a cell line that is biologically different from the parent cell line. While the adaptation process used herein may be supraphysiologic, the process did not result in a morphological change in the HNO cells. This biological difference, which is not noted morphologically, is also reflective of the clinical setting. The pathological definition of a tumor subtype only crudely predicts the biological state of that tumor and does not reflect its sensitivity to a given

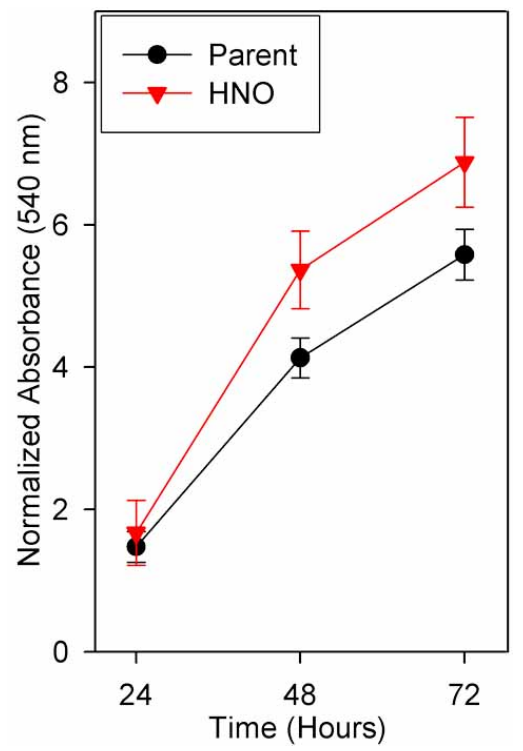

Fig. (5). MTT proliferation/viability assays of A549 parent and A549-HNO cells in serum-less media. The media of A549-HNO was supplemented with $600 \mu \mathrm{M}$ DETA-NONOate.

treatment. Therefore, we hypothesize that these cell line pairs will serve as a valuable model to determine the molecular differences between low and HNO adapted cells, and how they respond to various treatments.

Due to the difficulties in adapting the LP07 cells to high concentrations of DETA-NONOate, it is unknown whether the cells would have been able to reach the same $600 \mu \mathrm{M}$ plateau as the A549 cells, had the adaptation process been carried out for a longer period of time. However, the endpoint verification experiment confirmed that we carried out the LP07 adaptation to a nitric oxide level that was sufficiently high enough to invoke a biological change in the 

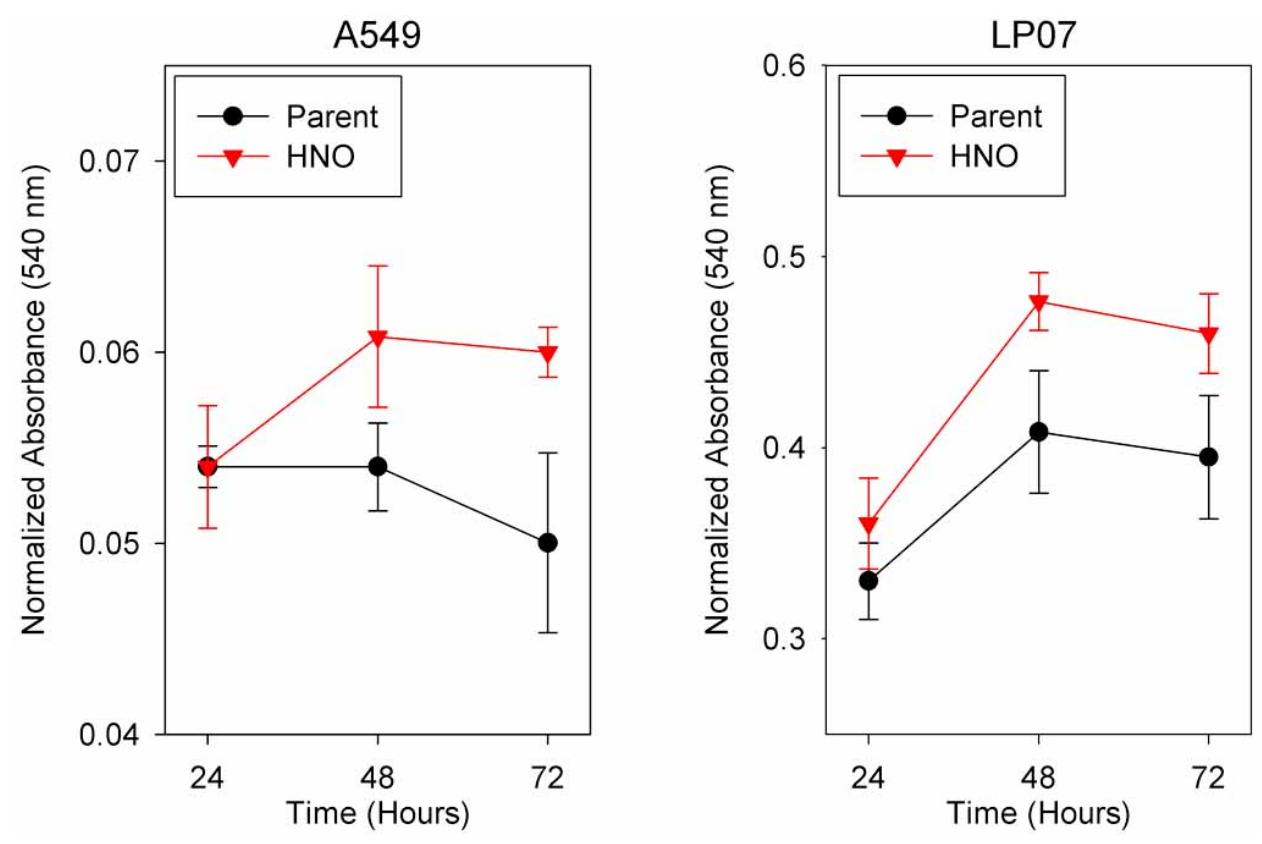

Fig. (6). MTT proliferation/viability assays of A549 and LP07 parent and HNO cells on soft agar. The media of the HNO cells was supplemented with $300 \mu \mathrm{M}$ (LP07-HNO) or $600 \mu \mathrm{M}$ (A549-HNO) DETA-NONOate.

cells, i.e. $300 \mu \mathrm{M}$ is lethal to the parent LP07 cell line. Future studies will focus on determining the extent of the biological differences between the parent and adapted cell lines. It is interesting to note that LP07-HNO cells were not "cross" adapted to modest levels of $\mathrm{H}_{2} \mathrm{O}_{2}$ (Fig. 3), as were the A549-HNO cells, implying that LP07-HNO cells lack some protective mechanism that was not induced by the NO exposure. Future molecular studies will be directed at elucidating this mechanism. In addition, future studies will be directed at using $\mathrm{H}_{2} \mathrm{O}_{2}$ to adapt cells to determine if the same molecular changes are induced. If so, this would be an important finding as it would broaden the versatility of this model system to reflect any free radical selective pressure encountered by tumors. It will also be of interest to see if the HNO cells are resistant to sulfur based free radicals, and if such exposure would make them NO resistant, as many fossil fuels produce reactive sulfur based components. Such a mechanism could explain how air pollution changes the nitric oxide biology of the upper aerodigestive tract and results in tumors arising in these tissues.

As mentioned previously, DETA-NONOate was selected for use in the adaptation process due to its favorable kinetic properties, most notably its longer half-life and higher delivery rate of NO relative to the other donors considered. The ability of the A549-HNO cells to survive and grow in the presence of other NO donors (Fig. 2) suggests that the cells have adapted to the NO produced by the donor, and not to any inherent toxicity resulting from exposure to the DETA-NONOate donor itself. Given this observation, we opine that the adaptation process would have been successful regardless of the donor chosen. However, we note that because the four donors considered (DETA-NONOate, spermine-NONOate, SNAP, and glyco-SNAP) possess different kinetic properties [22], the adaptation time and concentration (as defined here, in terms of the NO donor, and not in terms of the NO produced by the donor) required to reach an equivalent NO-environment would have varied.
Such differences in donor kinetics likely account for the different growth curves obtained in Fig. (2) between the NONOate and thiol-based donors. The NONOate donors (DETA-NONOate and Spermine-NONOate) contribute two moles of NO per mole of donor, while the thiol donors contribute only one. Thus, the decreased viability of the A549-HNO cells in the presence of the NONOate donors, relative to the thiol donors, was likely a result of the higher free-radical environment created by the additional NO generated. In reality, the kinetics of adaptation is not important. Patients that smoke, for example, may vary their exposure and amount to cigarette smoke over time. Some of these patients will present with clinical tumors earlier and with fewer pack-years, than other patients. The difference between these patients may not only be the differences in their exposure levels, but also in their biological constitution, as noted by the differences seen between the two cell lines used herein.

Our previous studies found that the mode and kinetics of NO delivery (i.e., the identity of the NO donor) strongly impacted the potential for genotoxicity and apoptosis at the enzymatic and gene expression levels of lung tumor cell lines following short-term exposure to NO donors [21,22]. At this time it is impossible to make similar conclusions about the biological properties of the adapted cells prepared in this study for a number of reasons: 1) Only one donor, DETA-NONOate, was used in the adaptation process, 2) In contrast to previous studies, the cells in the current experiment were exposed to donor for long periods of time, thus likely affecting additional and different biological pathways than are affected by short-term exposure, and 3) We have not yet identified the biological alterations arising within the HNO cells. Regardless of the donor chosen, however, we believe the cyclical nature of the NO concentration exposed to the cells during adaptationrepeated treatment of the cells with fresh media containing donor, followed by periods of donor decay—likely serves as 
a good in vitro method to mimic the cyclical exposure to nitrogen and oxygen reactive species that many lung cancer patients receive (for example, from long-term, periodic tobacco use). Furthermore, the successful adaptation of the LP07 mouse adenocarcinoma cell line suggests that we will be able to use these HNO cell lines in future in vivo animal models. It remains to be determined if the application of low dose, long-term exposure of any NO donor could result in in vivo tumor formation.

The results of the MTT assays (Figs. 4-6) indicate the adapted cells exhibit more aggressive growth than the parent cells under both normal growth and harsh conditions. These results suggest that we have successfully developed a model system consisting of two extreme cell types: 1) the parent cells, which are able to survive and grow in low-NO environments with nutrient supplements, and 2) the HNO cells, which represent a stronger tumor capable of growing robustly in a high-free radical environment, and other harsh conditions. The adaptation process carried out in this study further illustrates how such a prolonged, gradual increase in exposure to reactive nitrogen and oxygen species might transform a tumor originally only able to survive in a low$\mathrm{NO} /$ nutrient environment into one that is able to survive and grow in a harsh, high free-radical environment. This observation offers tremendous support to the current belief that nitric oxide plays a key role in tumor progression.

This is the first time that cell lines have been "adapted" to such high levels of nitric oxide. Moreover, studies carried out to date (including those from our own laboratory $[21,22]$ ) have typically only investigated short-term exposure to high levels of NO donor, rather than the slow adaptation approach used here. Given the simplicity in which the adaptation process was carried out in the current study, we believe that our adaptation method can be easily extended to the study of other cell lines, as well as to other chemical stimuli affecting cellular function. It will be important to undertake these studies to prove that this is a universal biological property not only between different cell lines of origin, but also between various pathological subtypes arising within various tissues. Defining the molecular changes within this matrix of cell types and tissue origins will provide new insights into NO tumor biology.

\section{CONCLUSION}

We have successfully adapted human and mouse lung tumor cells in increasing concentrations of the NO donor DETA-NONOate, resulting in a set of new, biologically different cell lines. The development of these HNO cell lines represents a significant finding unto itself, while also introducing a number of questions which will need to be addressed with future experiments. First, how do the HNO cell lines differ biologically from the parent cell lines at the molecular level? For example, do the HNO cell lines exhibit greater expression of oxidative stress proteins and/or other key cellular enzymes? Second, what happens when the NO source is removed indefinitely from the adapted cell line? Do the cells lose their ability to survive in the HNO environment, thereby reverting back to their original properties (i.e., the parental cell line), or is the ability to survive the harsh environment a permanent genetic change? Third, and most importantly, how do the properties of these
HNO cell lines correspond to tumors observed clinically, and how can the cell lines be used to better understand the clinical behavior of tumors? Do tumors growing in higher levels of nitric oxide need to be treated differently than tumors growing in lower levels on NO? Future experiments will be needed to answer these and many other questions.

In conclusion, while we do not yet know the extent of the biological differences between the HNO cell lines and their corresponding parent cell lines, we believe that the adaptation process outlined here represents a truly novel approach to studying the role of NO in human and mouse tumors. The HNO cell lines developed herein will serve as an important tool in future work aimed at understanding the role nitric oxide plays in the development and progression of lung cancer.

\section{ACKNOWLEDGEMENTS}

This work was supported by a VA Merit Review Grant (J.A.R.). The authors wish to thank Mr. Bill Paradise for his efforts in reviewing this manuscript.

\section{REFERENCES}

[1] American Cancer Society, Inc. Cancer facts \& figures 2008. Available at: http:/www.cancer.org/downloads/STT/2008CAFF finalsecured.pdf. Accessed March 23, 2009.

[2] Moller P, Folkmann JK, Forchhammer L, et al. Air pollution, oxidative damage to DNA, and carcinogenesis. Cancer Lett 2008; 266: 84-97.

[3] Koufman JA, Burke AJ. The etiology and pathogenesis of laryngeal carcinoma. Otolaryngol Clin North Am 1997; 30: 1-19.

[4] Bentz BG, Haines III GK, Radosevich JA. Increased protein nitrosylation in head and neck squamous cell carcinoma. Head Neck 2000; 22: 64-70.

[5] Ohshima H, Bartsch H. Chronic infections and inflammatory processes as cancer risk factors: possible role of nitric oxide in carcinogenesis. Mutat Res 1994; 305: 253-64.

[6] Thomas DD, Ridnour LA, Isenberg JS, et al. The chemical biology of nitric oxide: implications in cellular signaling. Free Radic Biol Med 2008; 45: 18-31.

[7] Jenkins DC, Charles IG, Thomsen LL, et al. Roles of nitric oxide in tumor growth. Proc Natl Acad Sci USA 1995; 92: 4392-6.

[8] Stuehr DJ. Enzymes of the L-arginine to nitric oxide pathway. J Nutr 2004; 134: 2748S-51S; discussion 2765S-67S.

[9] Knowles RG, Moncada S. Nitric oxide synthases in mammals. Biochem J 1994; 298: 249-58.

[10] Nathan C, Xie QW. Nitric oxide synthases: roles, tolls, and controls. Cell 1994; 78: 915-8.

[11] Bentz BG, Haines III GK, Lingen MW, Pelzer HJ, Hanson DG, Radosevich JA. Nitric oxide synthase type 3 is increased in squamous hyperplasia, dysplasia, and squamous cell carcinoma of the head and neck. Ann Otolaryngol Rhinol Laryngol 1999; 108: 781-7.

[12] Bentz BG, Haines III GK, Hanson DG, Radosevich JA. Endothelial constitutive nitric oxide synthase (ecnos) localization in normal and neoplastic salivary tissue. Head Neck 1998; 20: 304-9.

[13] Chandra R, Haines III GK, Bentz BG, Shah P, Robinson AM, Radosevich JA. Expression of nitric oxide synthase type 3 in reflux-induced esophageal lesions. Otolaryngol Head Neck Surg 2001; 124: 442-7.

[14] Ambs S, Bennett WP, Merriam WG, et al. Vascular endothelial growth factor and nitric oxide synthase expression in human lung cancer and the relation to p53. Br J Cancer 1998; 78: 233-9.

[15] Masri FA, Comhair SA, Koeck T, et al. Abnormalities in nitric oxide and its derivatives in lung cancer. Am J Respir Crit Care Med 2005; 172: 597-605.

[16] Lee TW, Chen GG, Xu H, et al. Differential expression of inducible nitric oxide synthase and peroxisome proliferatoractivated receptor gamma in non-small cell lung carcinoma. Eur J Cancer 2003; 39: 1296-301.

[17] Marrogi AJ, Travis WD, Welsh JA, et al. Nitric oxide synthase, cyclooxygenase 2, and vascular endothelial growth factor in the 
angiogenesis of non-small cell lung carcinoma. Clin Cancer Res 2000; 6: 4739-44.

[18] Liu CY, Wang CH, Chen TC, Lin HC, Yu CT, Kuo HP. Increased level of exhaled nitric oxide and up-regulation of inducible nitric oxide synthase in patients with primary lung cancer. $\mathrm{Br} \mathrm{J}$ Cancer 1998; 78: 534-41.

[19] Fujimoto H, Sasaki J, Matsumoto M, et al. Significant correlation of nitric oxide synthase activity and p53 gene mutation in stage I lung adenocarcinoma. Jpn J Cancer Res 1998; 89: 696-702.

[20] Chen GG, Lee TW, Xu H, et al. Increased inducible nitric oxide synthase in lung carcinoma of smokers. Cancer 2008; 112: 372-81.

[21] Bentz BG, Hammer ND, Radosevich JA, Haines III GK. Nitrosative stress induces DNA strand breaks but not caspase mediated apoptosis in a lung cancer cell line. J Carcinog 2004; 3: 16.

[22] Bentz BG, Hammer ND, Milash B, et al. The kinetics and redox state of nitric oxide determine the biological consequences in lung adenocarcinoma. Tumor Biol 2007; 28: 301-11.

[23] Lieber M, Smith B, Szakal A, Nelson-Rees W, Todaro GJ. A continuous tumor-cell line from a human lung carcinoma with properties of type II alveolar epithelial cells. Int J Cancer 1976; 17: $62-70$.

[24] Giard DJ, Aaronson SA, Todaro GJ, et al. In vitro cultivation of human tumors: establishment of cell lines derived from a series of solid tumors. J Natl Cancer Inst 1973; 51: 1417-23.

[25] Parma M, Diament MJ, Garcia C, Piccinni E, Mondelo N, Klein S. Mechanisms of paraneoplastic syndromes in mice bearing a spontaneous lung adenocarcinoma. Tumor Biol 1999; 20: 304-11.

[26] Urtreger AJ, Diament MJ, Ranuncolo SM, et al. New murine cell line derived from a spontaneous lung tumor induces paraneoplastic syndromes. Int J Oncol 2001; 18: 639-47.

[27] Diament MJ, Garcia C, Stillitani I, et al. Spontaneous murine lung adenocarcinoma (p07): a new experimental model to study paraneoplastic syndromes of lung cancer. Int J Mol Med 1998; 2: 45-50.

[28] Ke N, Albers A, Claassen G, et al. One-week 96-well soft agar growth assay for cancer target validation. Biotechniques 2004; 36: 826-3.

(C) Radosevich et al.; Licensee Bentham Open .

This is an open access article licensed under the terms of the Creative Commons Attribution Non-Commercial License (http://creativecommons.org/licenses/by-nc/ 3.0/) which permits unrestricted, non-commercial use, distribution and reproduction in any medium, provided the work is properly cited 